\title{
The role of steroid treatment in intractable cystitis glandularis: A case report and literature review
}

\author{
Ozgur Haki Yuksel, MD; ${ }^{*}$ Ahmet Urkmez, MD; ${ }^{*}$ Tibet Erdogru, MD; ${ }^{\dagger}$ Ayhan Verit, MD; \\ *Department of Urology, Fatih Sultan Mehmet Research and Training Hospital, Istanbul, Turkey; 'Department of Urology, Memorial Atasehir Hospital, Istanbul, Turkey
}

Cite as: Can Urol Assoc J 2015;9(5-6):E306-9. http://dx.doi.org/10.5489/cuaj.2636 Published online May 13, 2015.

\section{Abstract}

Cystitis glandularis is a proliferative disease of the urinary bladder epithelium. It is rare in children. We report a case of a 23 -yearold female with intractable macroscopic hematuria and severe irritative bladder symptoms persisting for 13 years. The patient, who had undergone open and endoscopic bladder surgery at various medical centres, is currently being followed up at our clinic. Cystoscopy revealed multiple edematous papillary tumours on the bladder neck, trigone, and lateral wall on both sides and she underwent transurethral resection of the bladder tumour. The pathological diagnosis was cystitis glandularis in accordance with the histopathological reports obtained from the other medical centres. Her condition was resistant to transurethral resection, partial cystectomy, intravesical mitomycin, and bacillus Calmette-Guerin (BCG) treatment; it eventually could have affected the upper urinary tract. Oral steroid treatment was given for 6 months; after treatment, her symptoms improved and the cystoscopy revealed a dramatic improvement in her condition.

\section{Introduction}

Cystitis glandularis (CG) develops secondary to chronic obstruction or inflammation. ${ }^{1}$ It is a benign tumoral entity covered with columnar or cuboid epithelium with a cystic appearance characterized by proliferation of Von Brunn islets (transitional epithelium which invaginates into lamina propria, a process induced by irritation in chronic cystitis mimicking carcinomatous invasion). CG is characterized by goblet cells resembling those in the colonic epithelium and it can be combined with intestinal metaplasia. The etiology and treatment of CG have not been clarified yet. CG is rarely seen in children. ${ }^{2}$ Although CG is a benign tumoral entity of the bladder, it can cause symptoms, like hematuria and dysuria, and disrupts quality of life. As well as being symptomatic, CG lesions may affect the upper urinary tract by making neuroan- atomical changes in the bladder. Recurrence can be observed despite various treatment modalities. We report a case of CG, which was resistant to transurethral resection, partial cystectomy, intravesical mitomycin, and bacillus Calmette-Guerin (BCG) treatment and can affect the upper urinary tract. The patient's quality of life has been negatively affected due to bleeding and lower urinary tract symptoms (LUTS). We have also observed a dramatic response to oral steroid therapy.

\section{Case report}

A 23-year-old female came to our outpatient clinic with intermittent and repetitive episodes of macroscopic hematuria and irritative voiding symptoms for 13 years. Her general physical examination was unremarkable. She has been taking colchicine to treat familial Mediterranean fever (FMF) since she was 14 (a MEFV gene mutation was present). When she was 16 , she had undergone cystoscopic examinations and cold punch biopsies several times because of her persistent macroscopic hematuria and LUTS. Histopathological examinations of the biopsy specimens revealed chronic cystitis, focal colonic metaplasia-CG, and ulcerative cystitis. She was followed up with palliative medical therapy.

Upon persistence of her complaints, urinary system ultrasonograms and abdominal tomograms were obtained when she was 17; these revealed a soft tissue mass extending from the posterior wall of the bladder into the bladder lumen. Open resection of the bladder mass was obtained, which was histopathologically reported as chronic inflammation, muscular hypertrophy, and CG. Subsequently, the patient received intravesical mitomycin and bacillus Calmette-Guerin (BCG) treatment. When she was 19, cold punch biopsy specimens were obtained under cystoscopic guidance and the histopathological report indicated chronic cystitis and focal intestinal metaplasia. The patient came to our clinic a year ago because of her persistent symptoms. Some of her biochemical test results were: $\mathrm{Hb} 7.4 \mathrm{gr} / \mathrm{dL}$, creatinine $0.7 \mathrm{mg} / \mathrm{dL}$, international normalized ratio 1.1, and her urine culture was unremarkable. She had macroscopic hematuria. She was a non-smoker. 
A contrast-enhanced magnetic resonance imaging (MRI) was administered (Fig. 1). Any pathology, except for an asymmetrically increased thickness of the posterior wall of the bladder, was not detected. Urinalysis did not disclose schistosoma parasites and 3-day urine tuberculosis culture was not pathologic. Cystoscopic examination performed under general anesthesia revealed refluxive and extremely dilated bilateral orifices. Under general anesthesia, her bladder capacity was $120 \mathrm{cc}$. On the bladder floor and contralateral wall, within an area of nearly $5 \mathrm{~cm}^{2}$, slightly elevated hemorrhagic suspect papillary lesions and telengiectasic bleeding foci on the rest of the bladder wall were detected (Fig. 2). Transurethral resection of bladder (TURB) was applied on the lesion. A histopathological examination of the specimen was reported as ulcerative active chronic cystitis and CG (Fig. 3). On subsequent video urodynamic examination, the bladder capacity was measured as $100 \mathrm{cc}$. Grade 3-4 reflux was detected bilaterally on a passive phase. On dimercaptosuccinic acid (DMSA) scanning, preservation of only $50 \%$ of the right and left renal functions was demonstrated. The patient was started on prednisolone (once daily doses of $10 \mathrm{mg}$ oral), propiverine $\mathrm{HCl}$ and prophylactic antibiotherapy. Improvement in symptoms was observed in the third month of treatment. In the second part of treatment, prednisolone $5 \mathrm{mg}$ per day was continued. Oral steroid treatment was given for 6 months; after treatment, her symptoms improved and the cystoscopy revealed a dramatic improvement in her condition (Fig. 4, parts A, B. Any adverse effect related to drug therapy was not observed. At the 6-month follow-up, her symptomatic improvement continued and her bladder capacity under general anesthesia was $250 \mathrm{cc}$. The patient has been followed up at 3 monthly intervals

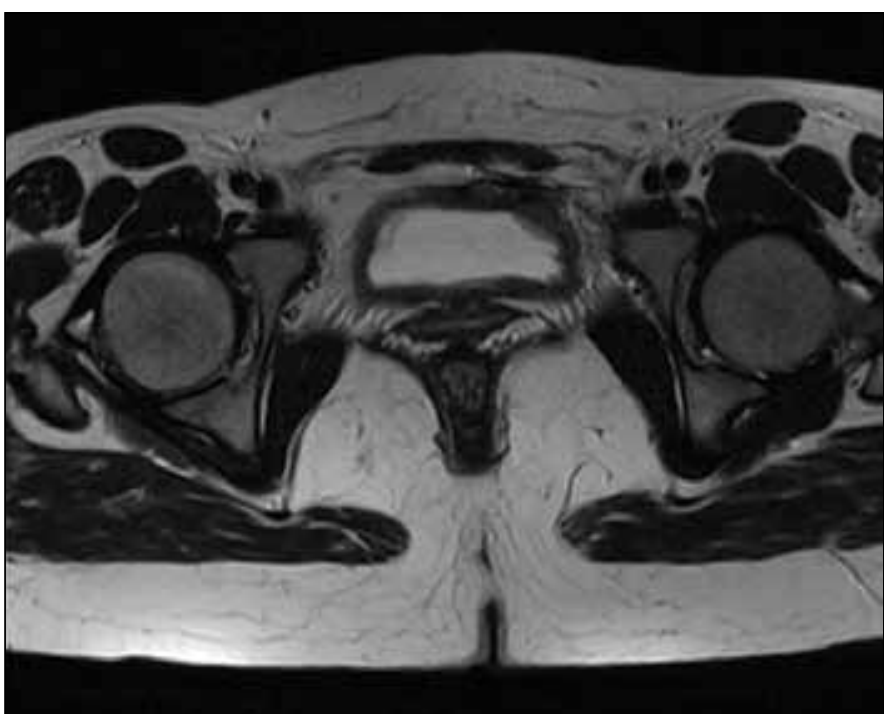

Fig. 1. Contrast-enhanced magnetic resonance imaging: On post-contrast sections, slightly enhanced contrast uptake is noted on the thickened posterior wall of the bladder. and voiding cystourethrogram (VCUG) and cystoscopy have been scheduled.

\section{Discussion}

Cystitis cystica and CG refer to invagination of the urothelium into the underlying lamina propria that have undergone cystic change or glandular metaplasia respectively. ${ }^{3,4}$ They are common lesions in $60 \%$ to $70 \%$ of autopsy cases ${ }^{5}$ and $0.1 \%$ to $1.9 \%$ in the clinical setting. ${ }^{6}$ They are most common in the trigone region of the bladder, but may be extensive. ${ }^{7}$ CG may occasionally appear as nodular, irregular masses on cystoscopic examination, mimicking a neoplasm.

Microscopically, two types are recognized: the usual type and intestinal type. Some of these lesions, especially the intestinal type, may mimic adenocarcinoma. ${ }^{8,9}$ In addition, the prognosis of CG is undetermined, with cases of widespread CG progressing to adenocarcinoma. ${ }^{3}$ This study was undertaken to evaluate the morphology of CG and to highlight the difference in morphology between CG and adenocarcinoma. A recent study found that CG was not a premalignant lesion and did not need frequent cystoscopic examination. ${ }^{10} \mathrm{CG}$ may be associated with chronic irritation of the bladder, following catheterization, stones, or inflammation. ${ }^{2}$ Widespread CG is seen in bladder extrophy, ${ }^{2}$ neurogenic bladder, ${ }^{11}$ and in association with pelvic lipomatosis. ${ }^{5}$ Among other reasons, avitaminosis, allergy, hormonal imbalance, carcinogenic drugs and IgA-mediated immune mechanisms can be enumerated. CG is present in $14 \%$ to $67 \%$ of patients with non-urachal adenocarcinoma of the bladder, ${ }^{12}$ while CG with atypia in a fundal-based adenocarcinoma excludes the diagnosis of urachal adenocarcinoma. ${ }^{13}$ Therefore in these cases, endoscopic evaluation is recom-

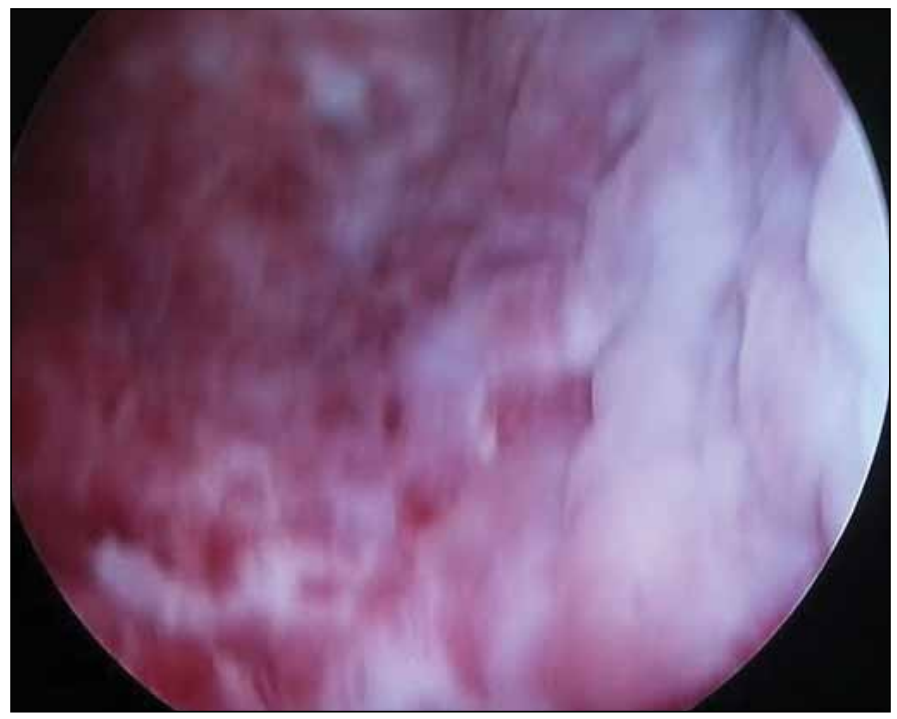

Fig. 2. Cystoscopic image before oral steroid treatment. 


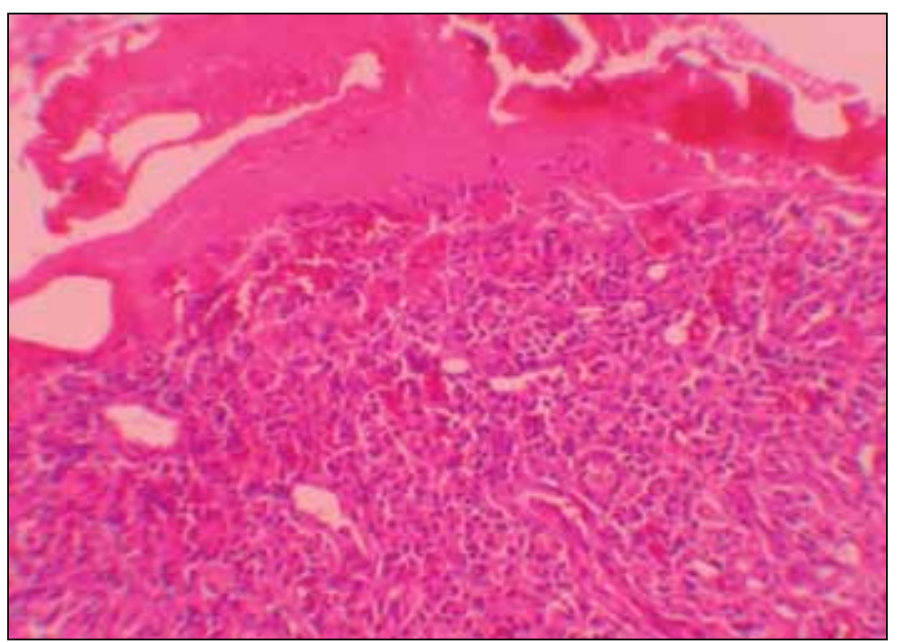

Fig. 3. Histopathologic image: Fibrinopurulent exudate (arrow) on the surface, diffuse inflammatory cells within the bladder wall.

mended. Treatment consists of transurethral resection and elimination of inflammation or obstruction. As seen in our case, when we reviewed the literature on the treatment of recurrent CG and repetitive attempts of transurethral resections, we found that optimal doses of BCG, ${ }^{14}$ intravesical instillation of chemotherapeutic agent (pirarubicine),$_{1}^{15}$ oral steroid therapy, ${ }^{6}$ or intravesical steroid application ${ }^{16}$ and YAG: laser ablation ${ }^{17}$ were administered. Cystectomy with urinary diversion for CG is rare. ${ }^{18-20}$

It is believed that CG is due to chronic irritation to the bladder epithelium and does not usually cause symptoms.
CG may cause irritative symptoms, hematuria, and rarely hydronephrosis. ${ }^{7,21-23}$ In a recent study, results showed that CG accompanied with upper urinary tract obstruction shares the same pathological feature and immune profile as CG without upper urinary tract obstruction. No significant differences of immune histochemical expression in tissue are found in CG with different pathological types. ${ }^{24}$

There is no standard treatment for oral steroid therapy for CG. The protocols of almost all steroid treatments for any disease are controversial. As mentioned, there are several treatment methods for recurrent lesions following endoscopic intervention. In our case, despite recurrent open and transurethral resection, intravesical BCG therapy and chemotherapy, irritative voiding symptoms persisted.

\section{Conclusion}

We studied therapy alternatives for our patient with CG since she was resistant to intravesical treatments and her symptoms were getting worse. Thus, intravesical steroid treatment was skipped with the patient's permission. She was given steroid treatment orally for 6 months and after this treatment; after treatment, her symptoms improved and the cystoscopy revealed a dramatic improvement in her condition. Oral steroid therapy is a good alternative for patients resistant to other treatments despite its adverse effects.

Competing interests: The authors declare no competing financial or personal interests.

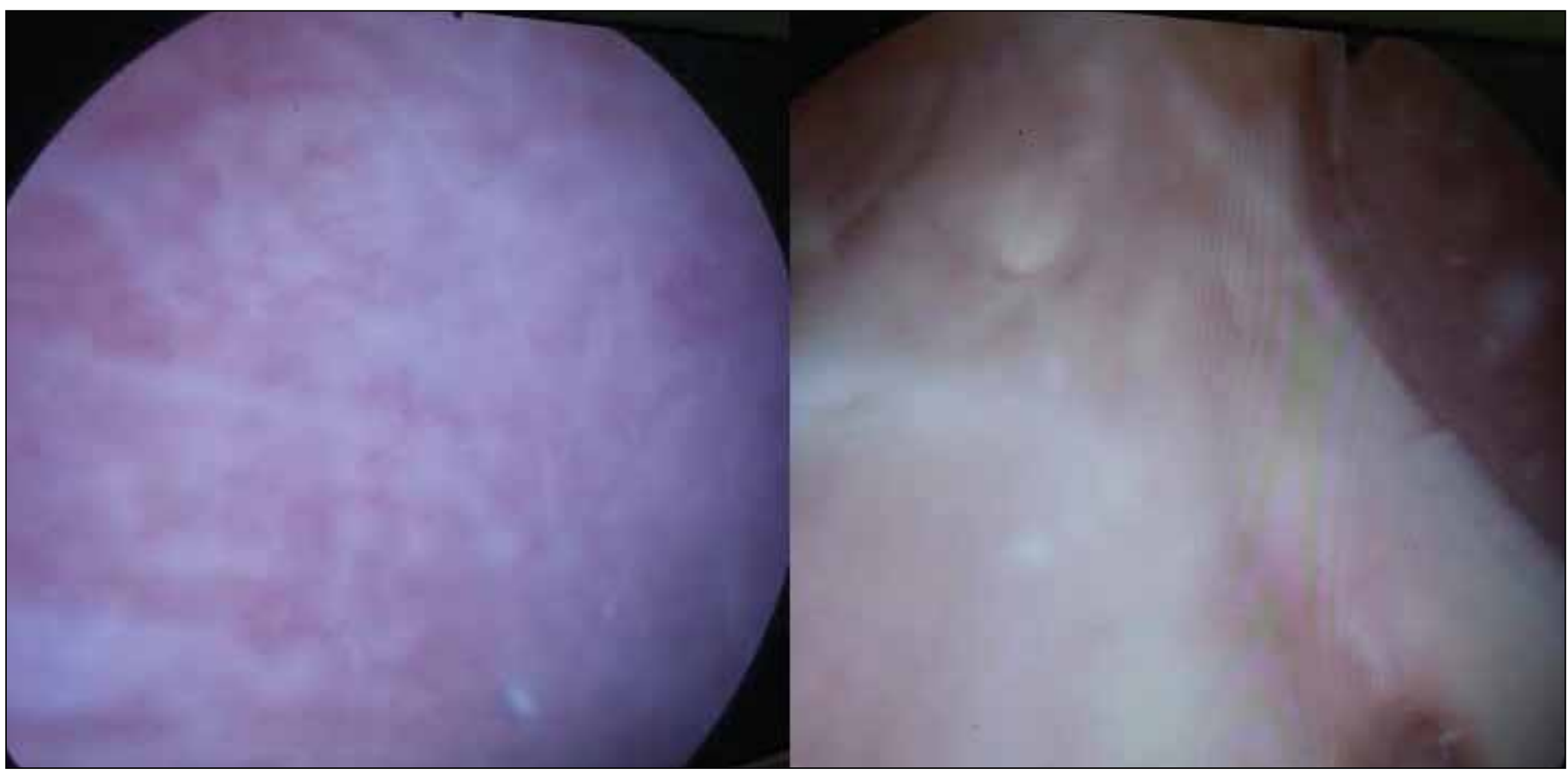

Fig. 4. Cystoscopic image after oral steroid treatment. 
This paper has been peer-reviewed.

\section{References}

1. Semins MJ, Schoenberg MP. A Case of florid cystitis glandularis. Nat Clin Pract Urol 2007;4:341-5. http://dx.doi.org/10.1038/ncpuro0814

2. Capozza N, Collura G, Nappo S, et al. Cystitis glandularis in children. BJU Int 2005;95:41 1-3. http:// dx.doi.org/10.1111/i.1464-410X.2005.05311.x

3. Smith AK, Hansel DE, Jones JS. Role of cystitis cystica et glandularis and intestinal metaplasia in development of bladder carcinoma. Urology 2008;71:915-8. http://dx.doi.org/10.1016/i.urology.2007.11.079

4. Srivastava S, Yan B, Chin SY, et al. Nuclear p53expression is associated with allelic imbalance (TP53) in glandular dysplasia and typical cystitis glandularis: A LCM-based molecular analysis. Clin Genitourin Cancer 2012;10:57-9. http://dx.doi.org/10.1016/i.clgc.2011.09.001

5. Rau AR, Kini H, Pai RR. Morphological evaluation of cystitis glandularis. Indian J Pathol Microbiol 2009;52:203-5. http://dx.doi.org/10.4103/0377-4929.48918

6. Lin $M E$, Huang $D$, Deng BH, et al. Expression and functional role of $\mathrm{Cdx} 2$ in intestinal metaplasia of cystitis glandularis. J Urol 2013;190:1083-9. http://dx.doi.org/10.1016/i.juro.2013.03.109

7. Maeda M, Hirabayashi $T$, Inuzuka $Y$, et al. A case of cystitis glandularis causing bilateral hydronephrosis. Nihon Hinyokika Gakkai Zasshi 2013;104:671-3. http://dx.doi.org/10.5980/ipniurol.104.671

8. Pantanowitz L, Otis CN. Cystitis glandularis. Diagn Cytopathol 2008;36:181-2. http://dx.doi. org/10.1002/dc.20774

9. Liu X, Chen Z, Ye Z. Etiological study on cystitis glandularis caused by bacterial infection. J Huazhong Univ Sci Technolog Med Sci 2007;27:678-80. http://dx.doi.org/10.1007/s1 1596-007-0615-y

10. Yi X, Lu H, Wu Y, et al. Cystitis glandularis: A controversial premalignant lesion. Oncol Lett 2014;8:1662-4. http://dx.doi.org/10.3892/ol.2014.2360

11. Delnay KM, Stonehill WH, Goldman $\mathrm{H}$, et al. Bladder histological changes associated with chronic indwelling urinary catheter. J Urol 1999;161:1106-8. http://dx.doi.org/10.1016/S0022-5347(01)61601-X

12. Thomas DG, Ward AM, Williams JL. A study of 52 cases of adenocarcinoma of the bladder. Br J Urol 1971;43:4-15. http://dx.doi.org/10.1111/j.1464-410X.1971.tb04925.x
13. Grignon DJ. Neoplasms of the urinary bladder. In: Bostwick DG, Elbe JN, editors. Urologic surgical pathology. St Louis: Mosby; 1997:215-305.

14. Mitre Al, Silveira CA, Leite KR, et al. Glandular cystitis: A rare benign condition presenting as a pseudo-tumor of the bladder. Clinics 2007;62:93-4. http://dx.doi.org/10.1590/S1807-59322007000100015

15. Li A, Liu S, Lu H, et al. Clinical character of cystitis glandularis accompanied with upper urinary tract obstruction. Can Urol Assoc J 2013;7:708-10. http://dx.doi.org/10.5489/cuai.359

16. Gómez dos Santos VG, Burgos Revilla FJ, García González R. Glandular cystitis. Endovesical steroid treatment. Arch Esp Urol 2000;53:461-4.

17. Stillwell TJ, Patterson DE, Rife CC, et al. Neodymium:YAG laser treatment of cystitis glandularis. J Urol 1988;139:1298-9.

18. Black PC, Lange PH. Cystoprostatectomy and neobladder construction for florid cysitis glandularis. Urology 2005;65:174. http://dx.doi.org/10.1016/i.urology.2004.07.026

19. Coelho RF, Marchini GS, Dall'oglio MF, et al. Cystoprostatectomy with ileal neobladder for treatment of severe cystitis glandularis in an AIDS patient. Clinics (Sao Paulo) 2008;63:713-6. http://dx.doi. org/10.1590/S1807-59322008000500023

20. Ye M, Wang WM, Zhu YJ, et al. Modified cystectomy with preservation of erectile and ejaculatory functions in men with nonmalignant bladder disease. Zhonghua Nan Ke Xue 2003;9:94-6.

21. Matsumura N, Hashimoto K, Katoh Y, et al. Three cases of proliferative cystitis causing hydronephrosis. Hinyokika Kiyo 2014;60:323-8.

22. Nariculam J, Patel A, Murphy D, et al. Cystitis cystica causing bilateral ureteric obstruction. Internet J Urol 2007;4:2.

23. Miglani U, Sinha T, Gupta SK, et al. Rare etiology of obstructive urophathy: Pelvic lipomatosis. Urol Int 2010;84:239-41. http://dx.doi.org/10.1159/000277606

24. Li A, Zhou J, Lu H, et al. Pathological feature and immunoprofile of cystitis glandularis accompanied with upper urinary tract obstruction. Biomed Res Int 2014;2014:872170. Epub 2014 May 29.

Correspondence: Dr. Ahmet Urkmez, Department of Urology, Fatih Sultan Mehmet Research and Training Hospital, Istanbul, Turkey; ahmeturkmez@hotmail.com 